\title{
A COLORING ALGORITHM FOR IMAGE CLASSIFICATION
}

\author{
D. GÓMEZ, J. MONTERO, J. YÁÑEZ AND C. POIDOMANI \\ Department of Statistics and O.R. \\ Complutense University \\ Madrid, Spain \\ E-mail:dagomez@estad.ucm.es
}

\begin{abstract}
In this paper we present a pixel coloring algorithm, to be considered as a tool in fuzzy classification. Such an algorithm is based upon a sequential application of a divisive binary procedure on a fuzzy graph associated to the image to be classified, taking into account surrounding pixels. Each color will suggest a possible class, if homogeneous, and the hierarchical structure of colors will allow gradation between classes.
\end{abstract}

\section{Introduction}

Classification in remotely sensing images quite often suggests techniques based upon fuzzy models. This is mainly the case when there are no objects to be classified. Objects, at least in a standard sense, use to present clear borders, and classification can be developed just based upon a boundary analysis and a previous knowledge of the shapes of the different objects under consideration. On the contrary, many classification problems about earth land use, for example, refer to classes showing gradation from one class to the next class. There are no clear boundaries, and each class defines a fuzzy set with no particular shape (see Bezdek and Harris ${ }^{6}$ ). In fact, there is an increasing research on Fuzzy Sets Theory applied to in remote sensing classification problems (see, e.g., Foody ${ }^{7}$ ).

Many different approaches can be found in remote sensing classification literature. In Amo et al. ${ }^{3,5}$, for example, some of the authors proposed a classification model based upon a modified outranking model, basically taken from Pearman et al. ${ }^{12}$. But the output information appeared to be difficult to be managed by non-qualified decision makers. A main need was to develop fuzzy representation techniques. In particular, it was missing some kind of coloring tool allowing a consistent and informative picture 
showing possible regions and gradation of membership to possible classes.

In this paper we propose an unsupervised crisp coloring methodology, to be considered within a more elaborated fuzzy classification system, as defined in Amo et $a^{2,4}$. The coloring procedure we present here is defined by means of a divisive crisp binary coloring process, which seems promising as a helpful tool in order to find out consistent regions and postulate possible fuzzy classes. In section 2 we introduce the basic pixels fuzzy graph associated to an image, and in section 3 we present a crude coloring algorithm. A final comments section shows the some particular improvements actually under development (see Gómez et $a .^{9}{ }^{9}$ ).

\section{The image and its associated pixels fuzzy graph}

Let us consider an image as a bidimensional map of pixels, each one of them being characterized by a fixed number of measurable attributes. These attributes can be, for example, the values of the three bands of the visible spectrum (red, green and blue), the whole family of spectrum band intensities, or any other family of physical measures.

Our main objective is to determine a family of pixels suggesting to define a class. This information should be taken into account in a later supervised analysis where additional information may exist.

The image $I$ under consideration is therefore divided into pixels ( $i n$ formation units), and the whole information is summarized as a vector of $b$ measures for each pixel $I=\left\{\left(x_{i, j}^{1}, \ldots, x_{i, j}^{b}\right) /(i, j) \in P\right\}$, where $P$ represents the associated set of pixels in which the image is divided, $P=\{(i, j) / i \in\{1, \ldots, r\} j \in\{1, \ldots, s\}\}$, meaning that we are dealing with an image of size $r \times s$, each pixel being characterized by $b$ numerical measures.

Given such an image $I$, a standard crisp classification problem pursues a partition in crisp regions, each one being a subset of pixels, to be considered a candidate for a new class, in case such a region is homogeneous enough. In this way, a crisp classification approach looks for a family of subsets of pixels $\left\{A_{1}, \ldots, A_{c}\right\}$ such that $P=\cup_{k=1}^{c} A_{k}$ but $A_{i} \cap A_{j}=\emptyset, \forall i \neq j$.

Our approach in this paper pursues to obtain an approximate gradation by splitting each subset under consideration into two crisp classes every time. The key tool will be a distance between the measured properties of pixels $d: P \times P \longrightarrow[0, \infty)$, which at a first stage can be based upon the Euclidean distance in $\mathbb{R}^{b}$. Of course, any other ad hoc distance can be taken into account in a future research. Obviously, the classification process will 
be strongly dependent on the selection of the appropriate distance, to be chosen taking into account all features of the image under consideration, together with our particular classification objectives.

Hence, our set of pixels $P$ is being modeled as a planar fuzzy graph (see, e.g., Kóczy ${ }^{10}$, Mordeson and Nair ${ }^{8}$, or Rosenfeld ${ }^{13}$ ) whose nodes are pixels, described by means of their cartesian coordinates $i \in\{1, \ldots, r\}$ and $j \in\{1, \ldots, s\}$. The graph will be planar in the sense that two pixels $(i, j)$ and $\left(i^{\prime}, j^{\prime}\right)$ cannot be linked if $\left|i-i^{\prime}\right|+\left|j-j^{\prime}\right|>1$. Consequently, two pixels could be adjacent only if they share one coordinate being the other one contiguous.

Let $\tilde{G}=(V, \tilde{E})$ be a fuzzy graph, where $V$ is the node set and the fuzzy edges set $\tilde{E}$ is characterized by the matrix $\mu=\left(\mu_{i j}\right)_{i, j \in V}$, where $\mu_{i j}=\mu_{\tilde{E}}(\{i, j\}), \forall i, j \in V$, and $\mu_{\tilde{E}}: V \times V \longrightarrow M$ is the associated membership function. Each element $\mu_{i j} \in M$ represents the intensity level of the edge $\{i, j\}$ for any $i, j \in V$.

The set $M$ is linearly ordered $\left(\mu_{i, j} \prec \mu_{i^{\prime}, j^{\prime}}^{\prime}\right.$ means that the intensity level of edge $\{i, j\}$ is lower than the intensity level of edge $\left.\left\{i^{\prime}, j^{\prime}\right\}\right)$. Hence, the set $M$ allows the literal graduation of the edge sets; for example, if $M=\{n, l, h\}$ the edges can be graduated as null $(n)$, low $(l)$ or high $(h)$.

We can then denote by $\widetilde{G(I)}=(P, \tilde{E})$ the graph associated to our image $I$, where $M=[0, \infty)$ is the domain of the distance function $d$ :

$$
\tilde{E}=\left\{\left\{d\left((i, j) ;\left(i^{\prime}, j^{\prime}\right)\right)\right\} \in F / \vee\left[\begin{array}{ll}
i=i^{\prime} & \left|j-j^{\prime}\right|=1 \\
j=j^{\prime} & \left|i-i^{\prime}\right|=1
\end{array}\right]\right\}
$$

Definition 2.1. Given the image $I$ and a distance $d$ between measured properties of pixels, the pixels fuzzy graph is defined as the pair $\widetilde{G(I)}=$ $(P, \tilde{E})$.

Notice that our pixels fuzzy graph $\widetilde{G(I)}$ can be also characterized by the set $P$ plus two $r \times s$ matrices, $D^{1}$ and $D^{2}$, where $D_{i, j}^{1}=d((i, j),(i+$ $1, j)), \forall(i, j) \in\{1, \ldots, r-1\} \times\{1, \ldots, s\}$ and $D_{i, j}^{2}=d((i, j),(i, j+1)), \forall \in$ $\{1, \ldots, r\} \times\{1, \ldots, s-1\}$. Since our coloring procedure will be based upon this alternative representation, from now on we shall denote our pixels fuzzy graph $\widetilde{G(I)}$ by $\left(r, s, D^{1}, D^{2}\right)$.

The key coloring algorithm proposed in the next section will take advantage of the above alternative representation, which shows relation between adjacent pixels in the pixels fuzzy graph $\widetilde{G(I)}$. 


\section{A crude coloring algorithm}

A $c$-coloring of a graph $G=(V, E)$ (see, e.g., Pardalos et al. $\left.{ }^{11}\right)$ is a mapping $C: V \longrightarrow\{1, \ldots, c\}$, verifying $C(v) \neq C\left(v^{\prime}\right)$ if $\left\{v, v^{\prime}\right\} \in E$. Any $c$-coloring induces a crisp classification of the nodes set $V$, being each class associated to one color: $V_{C}(k)=\{v \in V / C(v)=k\}, k \in\{1, \ldots, c\}$.

Our objective is to obtain a classification of pixels through a $c$-coloring $C$ of the pixels fuzzy graph $\widetilde{G(I)}$ : the pixel $(i, j) \in P$ will be classified as $k \in\{1, \ldots, c\}$ if its color is $C(i, j)=k$.

In order to color a fuzzy graph, we consider $G_{\alpha}$, the crisp graph defined by the $\alpha$-cut edge set $E_{\alpha}=\left\{\left\{e, e^{\prime}\right\} / \mu_{e, e^{\prime}} \geq \alpha\right\}$. The values of this parameter $\alpha$ will be selected in such a way that a successive binary coloring process will be applied to some fuzzy subgraphs of $\widetilde{G(I)}$.

The first binary coloring analyzes the pixels set $P$ classifying each pixel as 0 or 1 . The second binary coloring is applied separately to the subgraph generated by those pixels colored as 0 , to obtain the classes 00 and 01 , and to the subgraph generated by those pixels colored as 1 , to obtain the classes 10 and 11. This hierarchical process of binary coloring is repeated in the crude coloring process. In this way, a $c$-coloring $C$ will be defined on $\widetilde{G(I)}$ : if $C(i, j)=k$, with $k=6$ for instance, then the binary representation of $k-1=5$ is 101 , i.e. the pixel $(i, j)$ will be binary colored three times $(1$, 0 and 1 , respectively).

\subsection{The basic binary coloring procedure}

A natural way of introducing the basic binary coloring procedure is to classify two adjacent pixels as 0 and 1 if and only if the distance between them is greater or equal than a prescribed threshold $\alpha$. Notice that, in this way, only adjacent pixels are classified as distinct (if distance between them is high), while a standard approach classifies two arbitrary pixels in the same class if that distance is low (no matter they are adjacent or not).

Formally, and in order to define the first binary coloring procedure, given a value $\alpha$, let $G_{\alpha}$ denote the $\alpha$-cut of the fuzzy graph $\widetilde{G(I)}: G_{\alpha}=\left(P, E_{\alpha}\right)$, where

$$
E_{\alpha}=\left\{\{(i, j),(i+1, j)\} / d_{i, j}^{1}<\alpha\right\} \bigcup\left\{\{(i, j),(i, j+1)\} / d_{i, j}^{2}<\alpha\right\}
$$

The set $E_{\alpha}$ is the set of all pairs of adjacent pixels with a distance $d$ lower than $\alpha$.

Let $c o l: P \longrightarrow\{0,1\}$ be a binary coloring of $G_{\alpha}$. The first binary coloring can be obtained assigning an arbitrary color ("0" or "1") to certain 
pixel and fixing an order in which pixels will be colored. That first pixel to be colored could be, for example, the pixel $(1,1)$ in the left top corner of the image; then pixels will be colored from left to right and up to down depending on a fixed threshold $\alpha$. In general, given a colored pixel $(i, j)$ the adjacent pixels $(i+1, j)$ and $(i, j+1)$ will be subsequently colored. Since pixel $(i+1, j+1)$ can be alternatively colored either from pixel $(i+1, j)$ or from pixel $(i, j+1)$, a natural constraint is that both colors must be the same; otherwise, the coloring will be denoted as inconsistent.

Definition 3.1. Given a pixel set $P$ a square is a subset of four pixels

$$
s q(i, j) \equiv\{(i, j) ;(i+1, j) ;(i, j+1) ;(i+1, j+1)\}
$$

being $i \in\{1, \ldots, r-1\}$ and $j \in\{1, \ldots, s-1\}$.

We shall then denote by $P S$ the set of all squares, $P S=\{s q(i, j) / i \in$ $\{1, \ldots, r-1\}, j \in\{1, \ldots, s-1\}\}$.

Definition 3.2. Given a pixels fuzzy graph $\left(r, s, D^{1}, D^{2}\right)$, a square $s q(i, j) \in P S$ is consistent at level $\alpha$ if given an arbitrary color $\operatorname{col}(i, j)$, the above binary coloring procedure assigns the same color to pixel $(i+1, j+1)$, no matter if it is done from pixel $(i, j+1)$ or pixel $(i+1, j)$. Otherwise, the pixel square is inconsistent.

Consequently, the above binary coloring of pixels fuzzy graph depends on the chosen threshold value $\alpha$, and we have two extreme cases: $\bar{\alpha}=$ $\max _{(i, j) \in P}\left\{d_{i, j}^{1} ; d_{i, j}^{2}\right\}$ (if we fix a threshold $\alpha>\bar{\alpha}$, then the whole picture is considered as a unique class $(\operatorname{col}(i, j)=\operatorname{col}(1,1) \forall(i, j) \in P)$; and $\underline{\alpha}=$ $\min _{(i, j) \in P}\left\{d_{i, j}^{1} ; d_{i, j}^{2}\right\}$ (in case $\alpha<\underline{\alpha}$, the picture looks like a chess board, being all adjacent pixels alternatively classified as "0" and "1"). Only the interval $[\underline{\alpha}, \bar{\alpha}]$ should be properly considered.

Indeed, determining an appropriate intermediate $\alpha$ level is not a trivial task. But once a level $\alpha$ is given, the inconsistent squares can be detected with the binary function

$$
\text { inconsis }_{\alpha}\left(i, j, D^{1}, D^{2}\right)=\left\{\begin{array}{l}
1 \text { if } s q(i, j) \text { is inconsistent at level } \alpha \\
0 \text { otherwise }
\end{array}\right.
$$

where each value inconsis $s_{\alpha}\left(i, j, D^{1}, D^{2}\right)$ depends on the square values $d_{i, j}^{1}$, $d_{i, j+1}^{1}, d_{i, j}^{2}$ and $d_{i+1, j}^{2}$.

Pseudocode for computations relative to listing consistent and inconsistent squares, inconsis $\left(i, j, \alpha, D^{1}, D^{2}\right)$, has been developed by the authors in terms of four additional $0-1$ variables (see Gómez et al. ${ }^{9}$ ). 
Definition 3.3. Given a value $\alpha$, the pixels fuzzy graph $\left(r, s, D^{1}, D^{2}\right)$ is consistent at level $\alpha$ if all squares $s q(i, j) \in P S$ are consistent at level $\alpha$.

Definition 3.4. Given a pixels fuzzy graph $\left(r, s, D^{1}, D^{2}\right)$, its consistency level, denoted as $\alpha^{*}$, is the maximum value $\alpha \in[\underline{\alpha}, \bar{\alpha}]$ for which the fuzzy graph is consistent.

Existence of such a consistency level $\alpha^{*}$ is always assured, at least meanwhile our image contains a finite number of pixels. If some inconsistency is detected for a given $\alpha$ level, a decreasing procedure can be introduced in order to find a lower level $\alpha^{*}$ assuring consistency. Such a procedure will be initialized with $\alpha^{*}=\bar{\alpha}$, and then we search among inconsistent pixels $s q(i, j)$, by means of a new function newalpha (see Gómez et al. ${ }^{9}$ ). We can therefore look for a value $\alpha^{*}$ assuring consistency. Pixels are being classified either into a class "0" or a class "1" and in the next step we proceed to get a more precise color for both classes (class " 0 " will switch either into "00" or "01"). This will be done by alternatively activating only one of the classes already colored in a previous stage. Analogously, such a binary coloring process is applied in subsequent stages to those activated pixels under consideration (a subset of pixels $P^{\prime} \subset P$ ). This subset of pixels $P^{\prime}$ getting a more precise color at each stage can be also characterized by a matrix act such that $\operatorname{act}(i, j)=1, \forall(i, j) \in P^{\prime}$ and $\operatorname{act}(i, j)=0 \quad \forall(i, j) \notin P^{\prime}$.

We can compute the interval $[\underline{\alpha}, \bar{\alpha}]$ for the activated pixels, by means of a procedure initalpha $\left(r, s, D^{1}, D^{2}, a c t\right)$. It may be the case that two adjacent pixels are not activated, and therefore the process should stop. This situation can be easily detected in the associated pseudocode initalpha, where the lowest distance between activated pixels $\underline{\alpha}$ is initialized as a very big value (the greatest value of distances between activated pixels is initialized as 0$)$.

Again, notice that a given square can be consistent for a value $\alpha$ but inconsistent for another value $\alpha^{\prime}<\alpha$. Hence, a decreasing procedure must be repeated for the overall set $P S$ until we find a new level $\alpha^{*}$ assuring that all squares are made consistent in the new coloring environment.

Then, a function called consislevel is the core of our algorithm: it will iteratively compute the consistency level $\alpha^{*}$ for the family of pixels being actually activated (the initializing value will be $\bar{\alpha}$, which is obtained from the procedure initalpha). The input arguments of consislevel are the pixels fuzzy graph $\left(r, s, D^{1}, D^{2}\right)$ and the $r \times s$ matrix act. The interval $[\underline{\alpha}, \bar{\alpha}]$ is computed after the procedure initalpha is called, being $\alpha^{*}$ the returned value. The associated consislevel $\left(r, s, D^{1}, D^{2}\right.$, act) pseudocode computes 
the consistency level $\alpha^{*}(a c t)$, for a given a subset $P^{\prime} \subset P$ of activated pixels. Following the standard order in the activated pixels (at every stage) the level $\alpha^{*}(a c t)$ assures a valid binary coloring col procedure. In order to perform these computations, a procedure $\operatorname{bincol}_{\alpha}\left(r, s, D^{1}, D^{2}, a c t, c o l\right)$ will compute the binary coloring of activated pixels at level $\alpha$, the fist call to this procedure taking as initialization $\operatorname{col}(i, j)=0, \forall(i, j) \in P$ (see Gómez et $\left.a l .{ }^{9}\right)$.

\section{Final comments}

The final objective of the algorithm we propose in this paper is to show decision maker several possible pictures of the image, each one obtained by means of an automatic coloring procedure of each pixel based upon a particular distance. Such a coloring procedure takes into account behavior of each pixel with respect to its surrounding pixels, and each color will suggest a possible class. Our coloring process is based upon a basic binary procedure, which is again and again applied, leading to a hierarchical structure of colors (i.e., possible classes). This basic binary procedure evaluates the distance of the measurable description between adjacent pixels, assigning a color depending on whether such a distance is either lower or higher than a previously chosen threshold. Each colored picture can be analyzed by decision makers in a posterior classification procedure: certain homogeneous regions can be identified, and a subsequent comparison may lead to a fuzzy classification, if we are able to evaluate the degree of concordance of each pixel to each one of those identified regions (see Amo et al. ${ }^{1}$ ).

Due to space limitations, pseudocodes have not been included in this paper, but they can be obtained from the authors under request, plus additional details (see Gómez et al. ${ }^{9}$ ). Of course, the classification process induced by the previous binary coloring can be refined, and an appropriate relaxed coloring algorithm should be tried in order to bypass the computational inefficiency of the above crude coloring algorithm (see Gómez et al. ${ }^{9}$ for details). Once our basic binary coloring process has been successively applied $t$ times, we shall be able to distinguish $2^{t}$ classes. Our complete coloring process is therefore equivalent to a hierarchical classification procedure, obtaining as output a set of nested clusters, to be properly analyzed.

\section{Acknowledgments}

This Research has been partially supported by CICyT grant BFM20020281, Spain. 


\section{References}

1. A. Amo, D. Gómez, J. Montero and G. Biging: Relevance and redundancy in fuzzy classification systems. Mathware and Soft Computing 8, 203-216 (2001).

2. A. Amo, J. Montero and V. Cutello: On the principles of fuzzy classification. Proc. N.A.F.I.P.S. Conference, 675-679 (1999).

3. A. Amo, J. Montero and G. Biging: Classifying pixels by means of fuzzy relations. Int. J. General Systems 29, 605-621 (2000).

4. A. Amo, J. Montero, G. Biging and V. Cutello: Fuzzy classification systems. European Journal of Operational Research (to appear).

5. A. Amo, J. Montero, A. Fernández, M. López, J. Tordesillas and G. Biging: Spectral fuzzy classification: an application. IEEE Trans. Syst. Man and Cyb. (C) $32,42-48$ (2002).

6. J.C. Bezdek and J.D. Harris: Fuzzy partitions and relations: an axiomatic basis for clustering. Fuzzy Sets and Systems 1, 111-127 (1978).

7. G.M. Foody: The continuum of classification fuzziness in thematics mapping. Photogrammetric Engineering and Remote Sensing 65, 443-451 (1999).

8. J.N. Mordeson and S. Nair: Fuzzy graphs and Fuzzy Hypergraphs (PhysicaVerlag, Heidelberg, 2000).

9. D. Gómez, J. Montero, J. Yáñez and C. Poidomani: A fuzzy graph coloring algorithm for image classsification. Technical Report (Dept. Statistics and O.R., Complutense University, Madrid, Spain).

10. L. Kóczy: Fuzzy graphs in the evaluation and optimization of netwoks. Fuzzy sets and systems 46:307-319 (1992).

11. P.M. Pardalos, T. Mavridou and J. Xue: The Graph Coloring Problem: A Bibliographic Survey. In: D.Z. Du and P.M. Pardalos (Eds.): Handbook of Combinatorial Optimization, vol. 2 (Kluwer Academic Publishers, Boston, 1998); 331-395.

12. J. Montero, A. Pearman and J. Tejada: Fuzzy multicriteria decision support for budget allocation in the transport sector. TOP 3, 47-68 (1995).

13. A. Rosenfeld: Fuzzy graphs. In: L.A. Zadeh, K.S. Fu and M. Shimura (Eds.): Fuzzy sets and their applications to cognitive and decision processes (Academic Press, New York, 1975); 77-95. 